\title{
UMA CARACTERIZAÇÃO DE MEDIDAS DE DESEMPENHO PARA AVALIAR O POSTPONEMENT: ESTUDOS DE CASO EM EMPRESAS DA INDÚSTRIA DE ALIMENTOS
}

\section{A CHARACTERIZATION OF PERFORMANCE MEASURES FOR MEASURING POSTPONEMENT STRATEGIES: CASE STUDIES IN FOOD COMPANIES}

\author{
Karine Araújo Ferreira* E-mail: karine@decea.ufop.br \\ Rosane Lúcia Alcântara Chicarelli** E-mail: rosane@dep.ufscar.br \\ Gisele Lorena Diniz Chaves*** E-mail: giselechaves@ceunes.ufes.br \\ *Universidade Federal de Ouro Preto, Ouro Preto, MG \\ **Universidade Federal de São Carlos, São Carlos, SP \\ ${ }^{* * *}$ Universidade Federal do Espírito Santo, Vitória, ES
}

\begin{abstract}
Resumo: O postponement, que consiste em retardar a configuração final e/ou movimentação de produtos até que os pedidos dos consumidores sejam recebidos, é uma estratégia cada vez mais utilizada por empresas preocupadas em gerenciar a complexidade e a variedade crescente de produtos. Apesar de 60 anos de investigação sobre o tema, pouco ainda se sabe sobre os resultados de sua aplicação, principalmente na indústria brasileira de alimentos, onde o conceito é ainda pouco discutido. Embora vários autores apontem diferentes medidas para avaliar o desempenho logístico e da cadeia de suprimentos, existem poucos trabalhos que retratem quais destas seriam medidas adequadas para avaliar os impactos do postponement. Assim, nesta pesquisa buscou-se investigar como a estratégia de postponement é aplicada em empresas da indústria de alimentos e quais as medidas de desempenho passíveis de serem usadas para avaliar os resultados dessa aplicação. Para atender estes objetivos, foram desenvolvidos seis estudos de caso em empresas do segmento de fabricação de sucos e conservas de frutas. Os resultados obtidos propiciaram confrontar as medidas de desempenho encontradas na literatura com as indicadas na pesquisa empírica e sistematizá-las de forma a obter uma possível relação de medidas para avaliação da estratégia de postponement.
\end{abstract}

Palavras-Chave: Medidas de desempenho. Postergação. Postponement. Indústria de alimentos.

\begin{abstract}
The postponement strategy is more and more used by companies with the increasing complexity and variety of products, currently required by the market. In essence, the postponement is used to delay as much as possible the final configuration and/or movement of products and services, until the demand known. Despite the increasing attention to the subject, little is known about is implementation, especially in the Brazilian food industry. This research aims to investigate how the postponement strategy is applied to food companies and what performance measures that can be used to evaluate the results of this application. To meet these goals were developed six exploratory case studies in orange juice and fruits processing companies. The results allow comparing the performance measures found in the literature with those obtained in empirical research and systematize them in order to obtain a list of performance measures for measuring postponement strategies.
\end{abstract}

Palavras-Chave: Performance measures. Postponement. Food companies. 


\section{INTRODUÇÃO}

O atual ambiente sócio-econômico mundial exige que as cadeias de suprimentos sejam capazes de se adaptar e responder rapidamente a mercados caracterizados por exigências como: menores custos, diferenciação de produtos, confiabilidade, redução dos prazos de entrega, melhoria no controle de qualidade e aumento da flexibilidade e da diversificação produtiva. Nesse sentido, a gestão da cadeia de suprimentos (supply chain management - SCM) tem-se caracterizado pela introdução de várias iniciativas e práticas que têm mudado a forma de se fazer e de se gerenciar os processos de negócio ao longo das cadeias de suprimentos (PIRES, 2009; FAÉ, 2007). Dentre as várias iniciativas e práticas verificadas para gestão da cadeia de suprimentos, merece destaque o postponement, que consiste em adiar o máximo possível a configuração final e lou movimentação de produtos e serviços, até que a demanda seja conhecida. Operacionalmente, a estratégia de postponement consiste em projetar e desenvolver produtos padrão ou genéricos que possam ser customizados rapidamente e a baixo custo, uma vez que a demanda seja conhecida. Envolve também a adoção de estratégias de estoque específicas, que permitam que esses produtos genéricos sejam estocados em diversos armazéns centralizados até o momento em que o pedido é realizado. Uma vez conhecida a demanda, o produto é deslocado para uma localização mais próxima ao cliente. Vários autores destacam o postponement como uma importante estratégia para lidar com a incerteza da demanda em ambientes turbulentos (PAGH e COOPER, 1998; VAN HOEK, COMANDEUR e VOSS, 1998; TWEDE, CLARKE e TAl, 2000).

Nos últimos anos, o presente tema tem despertado interesse de acadêmicos e executivos em diversos países. Desde que o conceito de postponement foi definido na literatura de marketing por Alderson em 1950, vários trabalhos abordando o assunto podem ser encontrados em diferentes áreas como logística, produção, projeto do produto e mais recentemente, na cadeia de suprimentos (YANG, BURNS e BACKHOUSE, 2004a). Apesar da atenção crescente ao tema, sua aplicação prática é ainda pequena e muitos estudos se limitam a revisões teóricas ou elaboração de modelos matemáticos e de simulação relacionados ao assunto. Adicionalmente, pouco ainda se sabe sobre os resultados de sua 
aplicação, principalmente na indústria brasileira de alimentos, onde o conceito é ainda pouco discutido. Uma das causas para o desconhecimento dos impactos desta estratégia se deve, dentre outros fatores, a falta de medidas para avaliação do desempenho do postponement (ZHANG e TAN, 2001). Embora vários autores apontem diferentes medidas para avaliar o desempenho logístico e da cadeia de suprimentos, existem poucos trabalhos que retratem quais destas seriam medidas adequadas para avaliar os impactos de estratégias como o postponement.

Assim, nesta pesquisa buscou-se investigar como a estratégia de postponement é aplicada em empresas da indústria de alimentos e quais as medidas de desempenho passíveis de serem usadas para avaliar os resultados dessa aplicação. Para atender os objetivos desta pesquisa, foram desenvolvidos seis estudos de caso exploratórios em empresas do segmento de fabricação de sucos e conservas de frutas, sendo que três delas têm como atividade principal o processamento do suco de laranja e as outras três, a fabricação de derivados de tomate. Foram realizadas entrevistas pessoais em cada empresa, principalmente com executivos das áreas de produção e logística.

Este artigo está estruturado em seis seções, incluindo o capítulo introdutório $\mathrm{Na}$ seção 2, são apresentados os principais conceitos e tipos de postponement. A seguir, a avaliação de desempenho do postponement é discutida na seção 3 , onde são destacadas as dimensões da medição de desempenho e as principais medidas para avaliar esta estratégia identificadas na literatura. O método de pesquisa adotado é discutido na seção 4. Os principais resultados dos estudos de caso são expostos na seção 5. Por fim, são apresentadas as considerações finais do trabalho (seção 6), e em seguida, as referências bibliográficas.

\section{POSTPONEMENT: DEFINIÇÃO E TIPOS}

O postponement é uma prática que está crescendo e sendo cada vez mais difundida na literatura acadêmica e em aplicações práticas. Inicialmente o conceito foi proposto por Alderson (1950) como uma maneira de mudar a forma, identidade ou lugar de produtos em um momento tão tarde quanto possível dentro dos processos de manufatura e distribuição física. O objetivo era reduzir riscos através 
da manutenção de produtos em uma localização central até o último momento possível ou em estado indiferenciado até o último ponto possível do fluxo de mercadorias. Assim, postergar a movimentação do produto foi denominado de "postponement de tempo" (time postponement), enquanto a postergação na diferenciação do produto foi denominada de "postponement de forma" (form postponement).

Em 1965, Bucklin estudou limites de aplicação da estratégia e criou o conceito oposto ao de postponement, o Princípio da Especulação (Principle of Speculation). Esse princípio é o inverso do postponement e consiste em finalizar as operações o mais cedo possível no processo de manufatura (BUCKLIN, 1965). Os trabalhos de Alderson e Bucklin sobre postponement foram visionários para seu tempo, porém os longos lead times na produção e na distribuição tornaram difícil a aplicação do conceito e não despertaram a merecida atenção empresarial na época. Depois de 1965, poucos trabalhos abordaram o assunto, e o tema foi retomado no final da década de 80 por Zinn e Bowersox (1988) que propuseram que o postponement poderia ser separado em cinco diferentes tipos, quatro relacionados com alterações de forma do produto (etiquetagem, embalagem, montagem e manufatura) e o quinto relacionado ao tempo (centralização dos estoques).

Bowersox e Closs (1996) definem dois tipos de postponement: o postponement de manufatura (ou forma) e o postponement logístico (de tempo). O postponement de manufatura consiste fabricar um produto base ou padrão em quantidades suficientes para realizar economia de escala, enquanto as características de finalização são adiadas até que os pedidos dos consumidores sejam recebidos. Já o postponement logístico consiste em manter toda linha de produtos já acabados em estoque centralizado. O deslocamento dos estoques é adiado até o recebimento do pedido dos clientes. Quando a demanda ocorre, os pedidos são transportados diretamente ao varejo ou ao consumidor.

Van Hoek (1999) conceitua postponement como uma maneira para atrasar ou postergar o ponto de diferenciação do produto, isto é, atrasar os processos em que os produtos são transformados em especificações únicas para o consumidor. Este mesmo autor define também, além do postponement de forma e tempo, o postponement de lugar. Para ele, o postponement de lugar implica em estocar os produtos acabados em localizações centrais, antes da demanda ocorrer (atrasando 
a determinação do local); enquanto o postponement de tempo consiste em atrasar a movimentação de produtos finais, efetuando-a apenas sob pedido (atrasando a determinação de tempo). Percebe-se que para Van Hoek (1999) o postponement logístico engloba o postponement de tempo e o de lugar, ou seja, a combinação de postponement de tempo e lugar é entendida como postponement logístico.

Já Pagh e Cooper (1998) combinaram quatro estratégias de postponement para a cadeia de suprimentos em uma matriz 2x2 (Figura 1). $\mathrm{Na}$ matriz, postponement de forma é chamado de postponement de manufatura (manufacturing postponement) e postponement de tempo é chamado de postponement logístico (logistics postponement). As quatro estratégias são então desenvolvidas pela combinação do postponement de manufatura e logística. As duas primeiras estratégias são representadas tanto pelo postponement de manufatura como pelo postponement logístico. As outras duas são formadas pela combinação de ambas, denominada de estratégia de postponement total, e pela ausência de ambas, denominada de estratégia de especulação completa.

Figura 1 - Matriz postponement especulação e estratégias genéricas da cadeia de suprimentos

\begin{tabular}{|c|c|c|c|}
\hline & \multicolumn{2}{|c|}{ LOGÍSTICA } \\
\hline & & $\begin{array}{c}\text { Especulação } \\
\text { Estoques descentralizados }\end{array}$ & $\begin{array}{c}\text { Postponement } \\
\text { Estoques centralizados } \\
\text { e distribuição direta }\end{array}$ \\
\hline \multirow{2}{*}{ 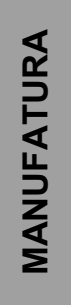 } & $\begin{array}{l}\text { Especulação } \\
\text { Fabricação para } \\
\text { Estocagem }\end{array}$ & $\begin{array}{l}\text { Estratégia de } \\
\text { especulação total }\end{array}$ & $\begin{array}{l}\text { Estratégia de } \\
\text { postponement de } \\
\text { logística }\end{array}$ \\
\hline & $\begin{array}{l}\text { Postponement } \\
\text { Fabricação por } \\
\text { Pedidos }\end{array}$ & $\begin{array}{l}\text { Estratégia de } \\
\text { postponement de } \\
\text { manufatura }\end{array}$ & $\begin{array}{c}\text { Estratégia de } \\
\text { postponement total }\end{array}$ \\
\hline
\end{tabular}

Fonte: Adaptado de Pagh; Cooper (1998, p.15).

Para incluir a aplicação do postponement em toda extensão da cadeia de suprimentos, Waller, Dabholkar e Gentry (2000) consideram mais apropriada a nomenclatura dada a partir dos estágios em que ele pode ocorrer, bem como seu envolvimento com os diferentes membros da cadeia de suprimentos. Assim, tais 
estratégias de postponement propostas pelos autores incluem o postponement upstream, postponement dowstream e postponement de distribuição. $\mathrm{O}$ postponement upstream (montante) ocorre com o retardo de ordens de materiais dos fornecedores até que se tenha informação de pedidos. É apropriado para empresas que empregam matérias-primas caras e para as que produzem a partir de projetos, como os construtores de reatores nucleares. Já postponement dowstream (jusante) é a postergação que agrega valor próximo da demanda e é executada pela empresa mais próxima do consumidor final (não necessariamente por quem produziu a primeira etapa). Este tipo de postponement pode ser definido como o atraso de algum tipo de mudança física no produto, após o estágio primário de manufatura, tais como, manufatura adicional, adição de características, ou adição de alguma função que adicione valor para uma cadeia de suprimentos especifica. Os autores mencionam também o postponement de distribuição (similar ao de lugar), que deve ocorrer depois que o produto já estiver com as configurações finais para ser consumido.

Cardoso (2002), Yang, Burns e Backhouse (2004b), Garcia-Dastugue e Lambert (2007) e outros autores de diferentes correntes literárias também propõem classificações de postponement, ampliando o leque de possibilidade. Em síntese, pode-se verificar que existem várias tipologias de postponement, envolvendo múltiplos elementos e diferentes aspectos. Isto é natural, uma vez que existem várias combinações possíveis de adiamento espaciotemporal e que trabalhos sobre o tema podem ser encontrados em diferentes correntes literárias.

O Quadro 1 sintetiza os tipos de postponement apresentados por cada autor. 
Quadro 1 - Síntese das classificações de postponement

\begin{tabular}{|c|l|}
\hline Autor & \multicolumn{1}{|c|}{ Classificações de Postponement } \\
\hline Alderson (1950) & $\begin{array}{l}\text { Postponement de forma } \\
\text { Postponement de tempo }\end{array}$ \\
\hline Zinn e Bowersox (1988) & $\begin{array}{l}\text { Postponement de forma é subdividido em 4 tipos: } \\
\text { de etiquetagem } \\
\text { de embalagem } \\
\text { de montagem } \\
\text { de fabricação } \\
\text { Postponement de tempo }\end{array}$ \\
\hline Bowersox e Closs (1996) & $\begin{array}{l}\text { Postponement de manufatura (= de forma) } \\
\text { Postponement logístico (= de tempo + de lugar) }\end{array}$ \\
\hline Van Hoek (1999) & $\begin{array}{l}\text { Postponement de forma } \\
\text { Postponement de tempo } \\
\text { Postponement de lugar }\end{array}$ \\
\hline Pagh e Cooper (1998) & $\begin{array}{l}\text { Especulação total } \\
\text { Postponement de manufatura (= de forma) } \\
\text { Postponement de logística (= de tempo + de lugar) } \\
\text { Postponement total }\end{array}$ \\
\hline Waller, Dabholkar e Gentry & $\begin{array}{l}\text { Postponement upstream } \\
\text { Postponement downstream (= postponement de forma) } \\
\text { Postponement de distribuição (= postponement de lugar) }\end{array}$ \\
\hline Cardoso (2002) & $\begin{array}{l}\text { Ausência de postergação } \\
\text { Centralizaçãa de estoques (= de lugar) } \\
\text { Diferenciação do produto (= de forma) } \\
\text { Combinações }\end{array}$ \\
\hline Yarcia-Dastugue e \\
Lambert (2007) & $\begin{array}{l}\text { Postponement de projeto do produto } \\
\text { Postponement de compras (= postponement upstream) } \\
\text { Postponement de produção (= de forma) } \\
\text { Postponement logístico (= de tempo + de lugar) }\end{array}$ \\
\hline $\begin{array}{c}\text { Postponement pela mudança na seqüência das atividades (= de } \\
\text { forma) } \\
\text { Postponement baseado no tempo (= de tempo) }\end{array}$ \\
\hline (2004b)
\end{tabular}

Fonte: Elaborado pelas autoras

Analisando as definições e classificações elaboradas em mais de 50 anos de estudo sobre o tema, é possível afirmar que a sua definição original ainda permanece atual, ou seja, apesar das diferentes conceituações e áreas de aplicação do conceito surgidas no decorrer dos anos, a idéia inicial proposta por Alderson em 1950 não sofreu grandes modificações. Neste trabalho também adotou-se esta classificação, ou seja, buscou-se investigar se as empresas analisadas adotam o postponement de forma e/ou tempo. Além dos tipos de postponement, foram identificadas nesta pesquisa em quais atividades o postponement acontece: operações e fabricação, manufatura final ${ }^{1}$, embalagem, etiquetagem, logística

\footnotetext{
${ }^{1}$ a manufatura final pode ser definida como algum tipo de mudança física no produto, após o estágio primário de manufatura.
}

Revista Produção Online. Florianópolis, SC, v.11, n. 2, p. 418-446, abr./jun., 2011 
(distribuição física); bem como qual o estágio da cadeia de suprimentos o postponement acontece.

De acordo com Cardoso (2002), estágio é o local da cadeia de suprimentos onde a diferenciação do produto pode acontecer. A mesma autora destaca os seguintes estágios da cadeia de suprimentos onde a postergação pode ser realizada: da planta fabril para um fornecedor upstream, entre plantas, de um depósito para uma planta fabril, de um cliente downstream para uma planta fabril ou para um depósito; e de depósito para depósito. É possível verificar que o postponement pode então ocorrer internamente em uma planta fabril ou ao longo de uma cadeia de suprimentos. No primeiro caso, adia-se somente o momento em que certas etapas da produção são realizadas, mantendo-se as operações produtivas no mesmo local, enquanto que, no segundo caso, as atividades de produção são postergadas para outros membros da cadeia de suprimentos, ou seja, a postergação pode ocorrer em locais diferentes de onde foram executadas as atividades de primárias de manufatura.

O Quadro 2 apresenta os tipos de postponement, atividades e estágios da cadeia de suprimentos que foram selecionados e serão analisados neste trabalho.

Quadro 2 - Classificações de postponement adotadas

\begin{tabular}{|c|c|c|}
\hline \multicolumn{2}{|c|}{ Classificações de postponement adotadas } \\
\hline $\begin{array}{c}\text { Tipos de } \\
\text { postponement }\end{array}$ & Atividade postergada & $\begin{array}{c}\text { Estágio da cadeia de } \\
\text { suprimentos }\end{array}$ \\
\hline $\begin{array}{c}\text { POSTPONEMENT DE } \\
\text { FORMA }\end{array}$ & $\begin{array}{c}\text { operães e fabricação } \\
\text { manufatura final } \\
\text { montagem } \\
\text { embalagem } \\
\text { etiquetagem }\end{array}$ & nontante (upstream) \\
POSTPONEMENT DE & nápria planta \\
TEMPO & justribuição & \\
\hline
\end{tabular}

Fonte: Elaborado pelas autoras

\section{AVALIAÇÃO DE DESEMPENHO DO POSTPONEMENT}

A medição de desempenho consiste em prover informações sobre as atividades executadas pela empresa, especialmente quando a variabilidade no desempenho exceder uma amplitude aceitável. Embora a medição de desempenho 
seja um tópico amplamente discutido, não existe uma única definição sobre o tema. A ampla literatura e a diversidade de situações onde a mensuração pode ser aplicada trazem uma variedade de definições. Uma das mais completas é a de Neely (1998, p.5) que define um sistema de medição de desempenho como um sistema que "permite que as decisões e ações sejam tomadas com base em informações, porque ele quantifica a eficiência e a eficácia das ações passadas por meio da coleta, exame, classificação, análise, interpretação e disseminação dos dados adequados".

A eficiência e efetividade de um sistema de medição de desempenho podem ser determinadas por um conjunto de medidas ou indicadores de desempenho. Segundo Lima Jr. (2001), as medidas de desempenho possibilitam que as avaliações sejam feitas com base em fatos, dados e informações, o que dá maior confiabilidade às conclusões. São relações matemáticas, medidas quantitativas e qualitativas de um processo ou de um resultado e estão, em geral, associados a uma meta qualquer.

Assim como a definição de medição de desempenho, também não existe na literatura uma definição única sobre as melhores medidas / indicadores de desempenho para avaliar a estratégia de postponement, ou qualquer outra. A revisão bibliográfica nos principais periódicos mundiais sobre o assunto realizado por Chow, Heaver e Henriksson (1994), cobrindo o período de 1982 a 1992, revelou as várias definições de avaliação de desempenho e indicadores existentes sobre o tema. Isso é resultado de diferentes interesses dos pesquisadores e da complexidade do desempenho que vem sendo aludida há um bom tempo. Apesar de pesquisadores e práticos terem como propósito obter um conjunto de indicadores que, coletivamente, alcancem a maior parte possível, se não toda, das mais importantes dimensões do desempenho a longo e curto prazo, uma abordagem sistemática geral para medição de desempenho ainda não foi desenvolvida. Isto ocorre principalmente devido a características de medidas específicas requeridas por cada organização (BEAMON, 1999).

A seguir, apresenta-se uma breve contextualização sobre as dimensões da medição de desempenho e qual delas será o foco deste trabalho. Em seguida, as 
principais medidas encontradas na literatura para avaliar o postponement são discutidas.

\subsection{Dimensões da medição de desempenho}

Segundo Neely, Gregory e Platts (2005) apud Mergulhão (2007), a medição de desempenho pode ser vista por três diferentes níveis. Estes níveis, ilustrados na Figura 2, podem ser entendidos como uma estrutura imaginária, ou "constructo" teórico que permitem uma visão sistêmica da medição de desempenho (MERGULHÃO, 2007).

Figura 2 - Visão sistêmica da medição de desempenho

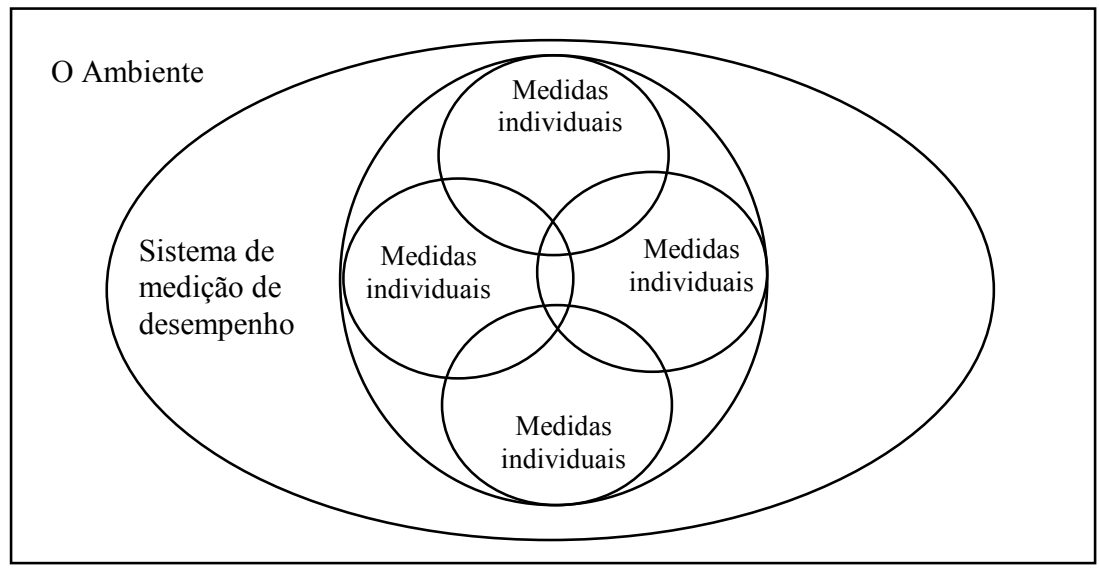

Fonte: Neely, Gregory e Platts (2005, p.1229).

Primeiramente, as medidas de desempenho são vistas individualmente como sendo ligadas aos objetivos e estratégia. Este seria o elemento fundamental da medição de desempenho. Depois, elas podem ser agrupadas de maneira a formar um conjunto de medidas de desempenho. Esse conjunto pode formar um sistema de medição de desempenho (SMD) desde que exista uma lógica para o agrupamento na escolha das medidas individuais. Por fim, o ultimo nível de analise é a interação do SMD com o ambiente organizacional, tanto interno quanto externo, de um sistema de operações. É importante ressaltar que associado à dimensão interna, o SMD deve ser consistente à cultura organizacional. Já para a dimensão externa, dois elementos devem ser destacados na elaboração das medidas de desempenho: os clientes e os competidores (NEELY, GREGORY e PLATTS 2005). 
A evolução da medição de desempenho pode ser dividida em duas fases distintas (GHALAYINI; NOBLE, 1996). A primeira fase relaciona-se ao período até os 1980s e é concentrada em medidas financeiras tais como o lucro, retorno no investimento e produtividade. A segunda fase, que começou no fim de $1980 \mathrm{~s}$ e continua até os dias atuais, corresponde à emergência de conceitos novos da gerência, tais como gestão da cadeia de suprimentos. Evidencia-se uma maior ênfase sobre a inclusão de medidas não financeiras e menos tangíveis, bem como necessidade de medidas de desempenho balanceadas (medidas financeiras e nãofinanceiras, além das de produtividade) e integradas para suportar as novas condições operacionais internas e externas da maioria das empresas. Segundo Saad e Patel (2006), o uso de métricas não financeiras é crucial para avaliar e promover a melhoria do desempenho de cadeias de suprimentos pelo apoio ao desenvolvimento de melhorias nos relacionamentos, na aprendizagem e em compromissos compartilhados segundo uma finalidade comum.

A segunda fase pode ainda ser desdobrada em duas novas fases, segundo Neely e Austin (2000). A primeira é a "miopia da medição", quando foi reconhecido que as empresas estavam medindo as coisas erradas. A segunda fase caracterizase pela "loucura da medição", quando as empresas são obcecadas com a medição e desejam medir tudo. Vale a pena destacar que ainda existem muitas empresas na primeira fase, ou seja, elas estão medindo coisas erradas.

Em função da inadequação da medição de desempenho tradicional relacionados à primeira fase de Ghalayini e Noble (1996), o uso balanceado de medidas financeiras e não-financeiras para refletir a complexidade das organizações torna-se um assunto de extrema importância. Para compensar essa lacuna, muitas estruturas de sistemas de medição de desempenho têm sido propostas na literatura.

Nesta pesquisa, estes sistemas de medição de desempenho e questões referentes a eles não foram detalhados. Devido à falta de estudos sobre medidas para avaliar o desempenho de empresas alimentícias, principalmente de estratégias como o postponement, o foco da pesquisa foi no primeiro nível de análise definido anteriormente por Neely, Gregory e Platts (2005). Assim, foram identificadas na literatura medidas de desempenho individuais adequadas para avaliar o desempenho do postponement em empresas alimentícias, confrontando-as com 
aquelas usadas no ambiente empresarial. Os resultados obtidos da pesquisa poderão servir como parâmetros para pesquisas futuras e elaboração de sistemas de medição de desempenho para avaliar o postponement.

\subsection{Medidas de desempenho para avaliar o postponement}

Uma estrutura de medidas de desempenho para avaliar o efeito do postponement é uma necessidade para mensurar a eficácia deste conceito. Uma vez que a implantação do postponement requer a re-configuração do produto e/ou o processo da cadeia de suprimentos, os dados históricos constituem uma fonte importante para validar a exatidão da implementação. As medidas associadas ao exame do impacto do postponement devem ser estudadas para avaliar corretamente sua aplicação. Por exemplo, atrasar as atividades de montagem final pode reduzir o custo de estoque em trânsito e o custo de manter estoque, mas por outro lado, pode aumentar a despesa total do processo de desenvolvimento do produto em curto prazo ou o custo de matérias-primas (FERREIRA, ALCÂNTARA e TOLEDO, 2006).

Vários autores propõem diferentes medidas de desempenho para avaliar os sistemas logísticos e a cadeia de suprimentos em diferentes dimensões, como os exemplos destacados no Quadro 3. 
Quadro 3 - Classificações das medidas de desempenho

\begin{tabular}{|c|c|c|}
\hline \multicolumn{3}{|c|}{ Classificações das Medidas de Desempenho } \\
\hline Tipo de medidas & $\begin{array}{l}\text { Dimensões em que as medidas } \\
\text { foram criadas }\end{array}$ & Autor (es) \\
\hline \multirow{3}{*}{ LOGÍSTICA } & $\begin{array}{l}\text { Logística interna } \\
\text { externa }\end{array}$ & $\begin{array}{l}\text { Lambert, Stock, Vantine (1998); } \\
\text { Quintão (2003) }\end{array}$ \\
\hline & $\begin{array}{l}\text { Gestão de estoques, transportes e } \\
\text { processamento de pedidos }\end{array}$ & Ferreira (2005) \\
\hline & $\begin{array}{l}\text { Serviço ao cliente, custos, gestão } \\
\text { de ativos e produtividade }\end{array}$ & Hijjar, Gervásio e Figueiredo (2005) \\
\hline \multirow{4}{*}{$\begin{array}{l}\text { GESTÃO DA } \\
\text { CADEIA DE } \\
\text { SUPRIMENTOS }\end{array}$} & Estratégica, tática e operacional & $\begin{array}{c}\text { Gunasekaran, Patel e Tirtiroglu } \\
\text { (2001) }\end{array}$ \\
\hline & $\begin{array}{l}\text { Fornecimento, fabricação, entrega, } \\
\text { retorno, geral }\end{array}$ & Sellito e Mendes (2006) \\
\hline & $\begin{array}{l}\text { Planejamento, } \\
\text { produção, entrega e aquisição, } \\
\text { (satisfação do consumidor) }\end{array}$ & Shepherd e Günter (2005) \\
\hline & $\begin{array}{l}\text { Cadeia de suprimentos direta e } \\
\text { reversa }\end{array}$ & $\begin{array}{l}\text { Mondragon, Lalwani e Mondragon } \\
\text { (2011) }\end{array}$ \\
\hline
\end{tabular}

Fonte: Elaborado pelas autoras

Apesar desses vários autores apontarem diferentes medidas para avaliar o desempenho das empresas, existem poucos trabalhos que retratem quais destas seriam medidas adequadas para avaliar os impactos do postponement. A revisão de literatura tem mostrado a falta de um sistema de medição de desempenho e poucos trabalhos identificam medidas para avaliar esta estratégia. Alguns autores, ao avaliarem o custo-benefício e resultados obtidos pela adoção do postponement apontam algumas medidas, focando em algum aspecto específico para sua avaliação, dentre eles: Zinn e Bowersox (1988); Lee e Billington (1994); Lee e Tang (1997); Van Hoek (1999).

Zinn e Bowersox (1988) desenvolveram um sistema para calcular e avaliar o custo direto e o benefício em um dado nível de serviço ao cliente, incluindo o custo de manutenção do estoque e custo de atividades como: rotulagem, embalagem, montagem e atividades de manufatura, além de custo de transporte e custo de vendas perdidas. Já Lee e Billington (1994) sumarizaram um conjunto de medidas de custo a serem utilizadas na implementação do postponement, que incluem: gestão de estoque, gestão de material, gestão de transporte, planejamento de 
projeto, gestão de fluxos reversos, prontidão organizacional, ajuste do ambiente externo, e as categorizam em mensuráveis e imensuráveis.

Lee e Tang (1997) desenvolveram um modelo para calcular o custo beneficio do postponement a um dado nível de serviço ao cliente, na qual inclui custo de projeto, custo de processamento, custo de manutenção de estoque e lead time. Van Hoek (1999) desenvolveu um sistema de medição, focando nos estágios de produção e distribuição onde o postponement ocorreu na cadeia de suprimentos da indústria de alimentos. O sistema compreendeu dois aspectos principais: eficiência e serviço do consumidor. As medidas identificadas são apresentadas no Quadro 4.

\section{Quadro 4 - Medidas de desempenho de eficiência e serviço ao cliente}

\begin{tabular}{|c|c|}
\hline \multicolumn{2}{|c|}{ Aspectos Avaliados } \\
\hline Eficiência & Serviço ao Cliente \\
\hline $\begin{array}{l}\text { Custo de mão de obra da manufatura } \\
\text { Custo de mão de obra da distribuição } \\
\text { Custo de transporte in-bound } \\
\text { Custo de distribuição física } \\
\text { Custo de armazenagem de bens semi- } \\
\text { acabados } \\
\text { Custo de armazenagem de bens acabados } \\
\text { Padronização de partes } \\
\text { Taxas de importação } \\
\text { Foco de no aumento da eficiência pela } \\
\text { centralização de estoque } \\
\text { Localização de certas atividades de } \\
\text { distribuição dentro do mercado Europeu }\end{array}$ & $\begin{array}{l}\text { Disponibilidade de produto } \\
\text { Lead time de entrega } \\
\text { Consistência, confiabilidade de entrega } \\
\text { Flexibilidade do sistema de produção } \\
\text { Aumento de responsividade para demandas } \\
\text { individuais do cliente } \\
\text { Acurácia e velocidade da informação ao } \\
\text { consumidor } \\
\text { Conveniência do pedido (eficiência, acurácia } \\
\text { e simplicidade) } \\
\text { Assistência na instalação (no local do } \\
\text { consumidor) } \\
\text { Aparência do produto } \\
\text { Amplitude da linha de produto oferecida } \\
\text { Número de novos produtos/ linha de produtos } \\
\text { oferecidas anualmente } \\
\text { Facilidade de retorno de produtos } \\
\text { Inovação do produto } \\
\text { Qualidade do produto } \\
\text { Qualidade da embalagem } \\
\text { Habilidade para mudar data de entrega de } \\
\text { produtos feitos sob pedido } \\
\text { Atendimento do pedido } \\
\text { Amplitude de preço da linha de produto } \\
\text { oferecida } \\
\text { Exigências de quantidade mínima de pedidos }\end{array}$ \\
\hline
\end{tabular}

Fonte: Adaptado de Van Hoek (1999)

O resultado da pesquisa de Van Hoek (1999) mostrou que custos de distribuição física foram importantes na medição de eficiência, enquanto os índices de entrega, serviço oferecido e inovação foram importantes na medição do serviço do consumidor. 
Apenas o trabalho de Zhang e Tan (2001) foi encontrado na literatura agrupando medidas de avaliação do postponement como um todo. Esses autores apresentaram uma estrutura de medidas de desempenho para o postponement que é baseada em Bowersox e Closs (1996), mas tem sido modificada para incorporar a discussão do postponement. Embora o trabalho de Bowersox e Closs (1996) tenha como foco principal a gestão logística, Zhang e Tan (2001) destacam que esta estrutura é adequada à avaliação do postponement pelos seguintes motivos: primeiro, o projeto e implementação do postponement afeta principalmente a função produção e a função logística, e conseqüentemente impacta a cadeia de suprimentos como um todo. Segundo, algumas estratégias de postponement, tais como postponement de lugar requerem a reconfiguração da rede de suprimentos. Terceiro, a estratégia de postponement é essencialmente uma estratégia de informação abrangendo a cadeia como um todo e possibilitando reduzir incertezas e melhorar a acurácia da previsão.

Os mesmos autores classificaram a medição do desempenho em custos internos e custos externos. Os custos internos são sub-divididos em quatro categorias (tipos): custo total, custo, serviço do consumidor e gestão de ativos, enquanto o custo externo compreende o custo ambiental, conforme pode ser visualizado na Figura 3.

Figura 3 - Categorias de medidas de desempenho para avaliar o postponement

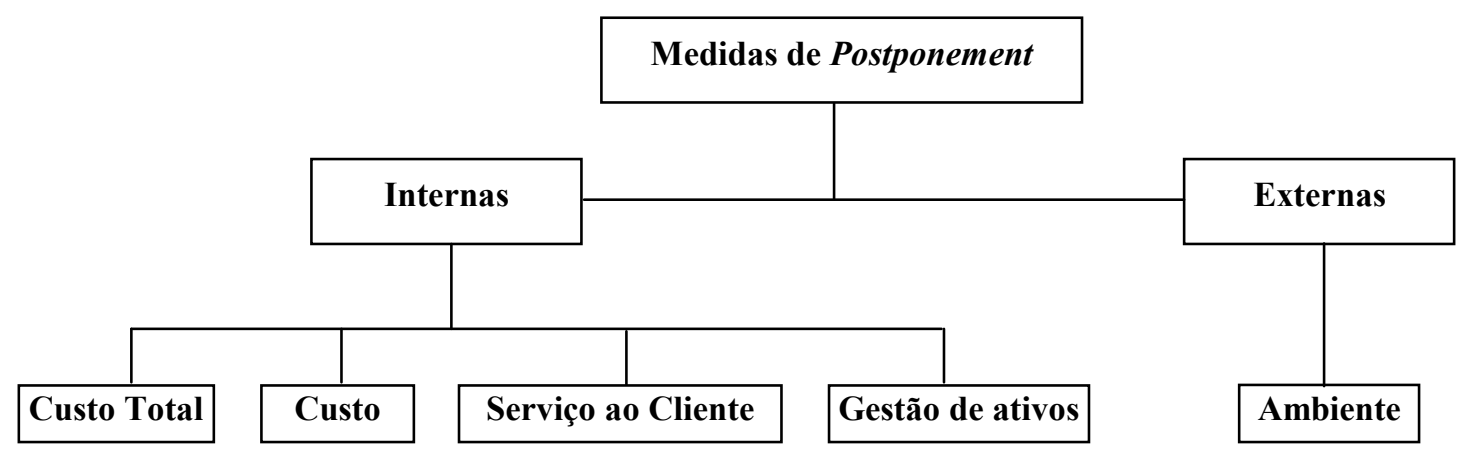

Fonte: Zhang e Tan (2001, p.52).

De acordo com as categorias destacadas na Figura 3, Zhang e Tan (2001) identificaram medidas de desempenho para avaliar o desempenho do 
postponement a partir de revisão bibliográfica sobre o tema, como mostra o Quadro 5.

Como o trabalho proposto por Zang e Tan (2001) foi o único estudo encontrado na literatura de postponement agrupando medidas de desempenho para avaliação desta estratégia, optou-se por adotar essas medidas de desempenho como base para realização desta pesquisa. Este trabalho foi selecionado por apresentar a revisão mais completa sobre medidas de desempenho para avaliação do postponement. Além disso, como as entrevistas foram realizadas em empresas processadoras de alimentos (empresa foco) e não em toda cadeia de suprimentos, optou-se por investigar apenas as medidas internas propostas por estes autores, ou seja, foram identificadas as medidas nas dimensões custo, gestão de ativos e serviço ao cliente. Na pesquisa realizada, outras medidas surgiram dentro destas dimensões e em outras, de acordo com o observado nas empresas analisadas. Assim, as medidas encontradas na literatura foram confrontadas com as indicadas pelas empresas participantes da pesquisa empírica.

Quadro 5 - Medidas de desempenho para avaliar o postponement

\begin{tabular}{|c|c|c|}
\hline Dimensão & Tipo & Medidas \\
\hline \multirow{23}{*}{ Interna } & \multirow[t]{3}{*}{ Custo Total } & Custo total \\
\hline & & Custo por unidade \\
\hline & & Custo total como uma porcentagem de vendas \\
\hline & \multirow[t]{10}{*}{ Custos Funcionais } & Custo de transporte \\
\hline & & Custo de armazenagem \\
\hline & & Custo no processo de rotulagem \\
\hline & & Custo no processo empacotamento \\
\hline & & Custo no processo de montagem \\
\hline & & Custo no processo de manufatura \\
\hline & & Processamento de pedidos \\
\hline & & Custo reverso \\
\hline & & Custo de matéria-prima \\
\hline & & Custo de mão de obra \\
\hline & \multirow[t]{5}{*}{ Serviço ao Cliente } & Taxa de entrega \\
\hline & & Taxa de falta de estoques por item \\
\hline & & Taxa de entrega no tempo \\
\hline & & Tempo de atendimento de pedidos em atraso \\
\hline & & Lead Time total \\
\hline & \multirow[t]{5}{*}{ Gestão de Ativos } & Giro de estoque \\
\hline & & Custo de manutenção de estoque \\
\hline & & Nível de estoque \\
\hline & & Retorno de investimento \\
\hline & & Retorno de ativos \\
\hline Dimensão & Tipo & Medidas \\
\hline \multirow{3}{*}{ Externa } & \multirow[t]{3}{*}{ Ambiente } & Taxas para o governo local \\
\hline & & Custo da matéria-prima local no produto final \\
\hline & & Custo da mão-de-obra local no produto final \\
\hline
\end{tabular}

Fonte: Adaptado de Zhang e Tan (2001).

Revista Produção Online. Florianópolis, SC, v.11, n. 2, p. 418-446, abr./jun., 2011 


\section{MÉTODO DE PESQUISA}

A pesquisa realizada neste trabalho é de abordagem qualitativa e o método adotado foi estudo de caso. Segundo Yin (2001, p.32), "um estudo de caso é uma investigação empírica que investiga um fenômeno contemporâneo dentro do contexto da vida real, especialmente quando os limites entre fenômeno e contexto não estão claramente definidos". O foco desta pesquisa é direcionado para a situação presente, ou seja, a amplitude que as empresas alimentícias têm utilizado o postponement e identificação das possíveis medidas de desempenho para avaliar os resultados da aplicação dessa estratégia.

Os estudos de caso desenvolvidos nesta pesquisa são de natureza exploratória, pois apesar de haver mais de 60 anos de estudos acadêmicos sobre o tema, o processo de adoção da estratégia de postponement no ambiente de negócio brasileiro e principalmente nas empresas de alimentos é ainda pouco explorado. Além disso, esta pesquisa foi desenvolvida em múltiplos casos, para aumentar o grau de validade externa e também reduzir qualquer viés do pesquisador no decorrer da pesquisa.

A unidade de análise para o estudo de caso é a empresa processadora de alimentos (setor industrial) que adote um ou mais tipos de postponement identificados na literatura. Foram então selecionadas para esta pesquisa seis empresas processadoras de alimentos, sendo três inseridas na sub-classe denominada "fabricação de sucos de frutas" e outras três, na sub-classe "fabricação de conservas de frutas". Estas empresas foram escolhidas, pois de acordo com a Classificação Nacional de Atividades Econômicas (2010), elas têm a maioria de seus produtos inseridos em uma mesma classe ou segmento denominado "fabricação de conservas de frutas, legumes e outros vegetais". Assim, essas empresas foram escolhidas como objeto de estudo por por atender aos seguintes requisitos da pesquisa: a) serem empresas processadoras de alimentos; b) adotarem algum tipo de postponement; c) estarem disponíveis para pesquisa; d) possuírem características comuns para efeito de comparação; e) serem representativas dentro deste segmento. 
Esta pesquisa foi conduzida por entrevistas semi-estruturadas, realizadas com os executivos responsáveis pelas áreas da produção ou logística, permitindo confrontação das respostas para maior consistência na análise. Foram utilizadas também nesta pesquisa fontes de dados adicionais como relatórios contendo informações sobre aplicação do postponement e observação direta, que foi realizada de maneira informal nas visitas realizadas nas empresas para coleta de dados.

\section{ESTUDOS DE CASO}

A seguir, são apresentados os resultados dos os estudos de caso realizados nas seis empresas do segmento de sucos e conservas de frutas.

\subsection{Caracterização das empresas}

As empresas $\mathrm{S1}$, S2 e $\mathrm{S} 3$ estão entre as quatro maiores empresas processadoras de suco de laranja no Brasil e estão situadas no estado de São Paulo. Já as empresas T1, T2 e T3 são empresas alimentícias que têm em comum a produção de derivados do tomate e estão entre as seis maiores empresas produtoras de tomate no Brasil. As empresas T1 e T2 estão localizadas no interior do estado de São Paulo e, a empresa T3, no interior de Goiás.

$\mathrm{Na}$ empresa $\mathrm{T} 1$, os derivados do tomate constituem a principal parcela dos produtos (70\%). Na empresa T2, a proporção é $40 \%$ de derivados de tomate e $40 \%$ de derivados de goiaba. Já na empresa T3, 30\% do total da sua produção é destinada aos derivados de tomate e 40\%, aos vegetais em conserva. Essas três empresas serão denominadas empresas produtoras de derivados do tomate, ou simplesmente, empresas do segmento de conservas de frutas. As principais características dessas empresas foram sintetizadas e são apresentadas no Quadro 6. 
Quadro 6 - Perfil das empresas do segmento de fabricação de sucos e conservas de frutas

\begin{tabular}{|c|c|c|c|c|c|c|}
\hline Características & Empresa S1 & Empresa S2 & Empresa S3 & Empresa T1 & Empresa T2 & Empresa T3 \\
\hline $\begin{array}{l}\text { Principais } \\
\text { Produtos }\end{array}$ & \begin{tabular}{|c|} 
Suco de \\
laranja \\
concentrado e \\
congelado \\
(SLCC); suco \\
integral \\
\end{tabular} & SLCC & $\begin{array}{l}\text { SLCC; suco } \\
\text { integral }\end{array}$ & $\begin{array}{c}\text { Derivados de } \\
\text { tomate }\end{array}$ & $\begin{array}{l}\text { Polpa e } \\
\text { derivados de } \\
\text { tomate e de } \\
\text { goiaba }\end{array}$ & $\begin{array}{l}\text { Derivados de } \\
\text { tomate e } \\
\text { vegetais em } \\
\text { conserva }\end{array}$ \\
\hline Outros produtos & \multicolumn{3}{|c|}{$\begin{array}{c}\text { óleo de laranja, essências e polpa cítrica, } \\
\text { aromas e fragrâncias, tintas, cosméticos, } \\
\text { complemento nutricional para ração } \\
\text { animal, etc. }\end{array}$} & $\begin{array}{l}\text { milho em } \\
\text { conserva, } \\
\text { ervilha, } \\
\text { geléias, } \\
\text { adoçantes, } \\
\text { etc. }\end{array}$ & $\begin{array}{l}\text { milho em } \\
\text { conserva, } \\
\text { achocolatado } \\
\text { em pó, } \\
\text { geléias, } \\
\text { mostarda, } \\
\text { etc. }\end{array}$ & $\begin{array}{c}\text { caldos, } \\
\text { sopas, } \\
\text { dressings } \\
\text { (maionese, } \\
\text { catchup e } \\
\text { mostarda) e } \\
\text { frutas em } \\
\text { calda, etc. }\end{array}$ \\
\hline Fundação & 1963 & 1991 & 1988 & 1955 & 1990 & 1955 \\
\hline $\begin{array}{c}\text { Unidades no } \\
\text { Brasil }\end{array}$ & 4 & 3 & 3 & 1 & 3 & 1 \\
\hline $\begin{array}{l}\text { Unidades } \\
\text { exterior }\end{array}$ & 1 & 0 & 2 & 0 & 0 & 0 \\
\hline $\begin{array}{l}\text { Número de } \\
\text { funcionários }\end{array}$ & 1700 & $\begin{array}{c}1800 \\
\text { (entressafra) } \\
3000 \text { (safra) } \\
\end{array}$ & \begin{tabular}{|c|}
400 \\
(entressafra) \\
3000 (safra) \\
\end{tabular} & \begin{tabular}{|c|}
350 \\
funcionários \\
diretos
\end{tabular} & $\begin{array}{c}960 \\
\begin{array}{c}960 \\
\text { funcionários } \\
\text { diretos }\end{array} \\
\end{array}$ & \begin{tabular}{|c|}
1300 \\
$\begin{array}{c}\text { funcionários } \\
\text { diretos }\end{array}$ \\
\end{tabular} \\
\hline Áreas de atuação & $\begin{array}{c}98 \% \\
\text { exportação }\end{array}$ & $\begin{array}{c}99 \% \\
\text { exportação }\end{array}$ & $\begin{array}{c}95 \% \\
\text { exportação }\end{array}$ & $\begin{array}{c}\text { Todo Brasil e } \\
\text { exportação }\end{array}$ & $\begin{array}{c}\text { Todo Brasil e } \\
\text { exportação } \\
(12 \%)\end{array}$ & $\begin{array}{c}\text { Todo Brasil e } \\
\text { exportação } \\
(8 \%)\end{array}$ \\
\hline $\begin{array}{l}\text { Parcela de } \\
\text { mercado }\end{array}$ & \multicolumn{3}{|c|}{$\begin{array}{l}\text { entre as quatro maiores processadoras } \\
\text { de suco de laranja do Brasil }\end{array}$} & \multicolumn{3}{|c|}{$\begin{array}{c}\text { entre as seis maiores processadoras de } \\
\text { derivados de tomate }\end{array}$} \\
\hline
\end{tabular}

Fonte: Elaborada pelas autoras com base nas entrevistas.

A seguir, a aplicação do postponement nestas empresas é discutida, seguida pela apresentação das possíveis medidas de desempenho para sua avaliação destacadas na pesquisa empírica.

\subsection{Aplicação do postponement nas empresas de sucos e conservas de frutas}

A estratégia de postponement foi verificada nas empresas investigadas, sendo observados tanto os tipos de postponement de forma como o postponement de tempo.

No caso das empresas processadoras de suco de laranja, as etapas de extração do suco da laranja são realizadas pelas empresas processadoras (empresas S1, S2 e S3) para estoque e em formulação praticamente única dos dois principais tipos de suco produzidos por estas empresas: suco de laranja 
concentrado e congelado; e suco de laranja integral (pronto para beber). O suco é então transportado até o país dos clientes, onde após a chegada ao destino, pode então ser customizado (geralmente por uma empresa cliente, responsável pelo processamento final e distribuição final do suco). Assim, algumas atividades de manufatura final, como mistura de diferentes tipos de sucos (também conhecido como processo de blendagem), diluição, adição de componentes responsáveis pelo sabor e aroma do suco, bem como adição de embalagem e rótulo são postergados para empresas engarrafadoras, geralmente localizadas no exterior, sendo realizadas somente no momento em que a demanda é mais conhecida. Já para as empresas produtoras de derivados de tomate, a matéria-prima (tomate) é pré-processada, transformada em polpa e, permanece armazenada no estado semi-acabado até que a demanda seja mais conhecida. Baseado nas especificações de receita e demanda por determinado tipo de produto final (purê, extrato, molho, etc), essa polpa é então transformada no produto final (derivados do tomate). Esse mesmo processo de postergação ocorre para os derivados da goiaba (doce de goiaba, goiabada cascão, goiabada em calda, etc) na empresa T2.

É importante destacar que embora o postponement de forma seja verificado nas seis empresas investigadas, este ocorre em diferentes atividades e locais na cadeia de suprimentos das empresas investigadas. No caso das empresas de suco de laranja, as atividades de manufatura final, como por exemplo: diluição, adição de componentes de sabor e aroma, além das atividades de envase, adição de embalagem e rótulo só ocorrem à jusante da cadeia de supriementos, sendo realizadas pelas empresas engarrafadoras, como por exemplo, a Tropicana. Para as empresas de derivados de tomate, as atividades geralmente são postergadas nas próprias unidades fabris, ou seja, internamente nas empresas T1, T2 e T3, atrasando somente o momento em que as diferenciações ocorrem.

Em relação ao postponement de tempo, percebe-se que este ocorre para todos os tipos de produtos nas empresas investigadas, não apenas para o suco de laranja, derivados de tomate e goiaba. Assim, os diferentes tipos de produtos são mantidos nos armazéns localizados nas unidades produtivas das empresas investigadas e são deslocados para seus destinos (empresas engarrafadoras, no caso das empresas processadoras de suco de laranja; e para os distribuidores, 
supermercados e demais canais de distribuição, no caso, das empresas produtoras de derivados do tomate e goiaba) somente após receber o pedido do cliente.

Em síntese, pode-se dizer que foi possível verificar que os tipos de postponement de tempo e forma são encontrados nas empresas de sucos e conservas de frutas investigadas, sendo que no caso do postponement de forma, este ocorre principalmente a partir das atividades de manufatura final nas empresas estudadas e não prioritariamente nas atividades de embalagem e rótulo como destacado por Van Hoek (1999) na literatura.

\subsection{Medidas de desempenho para avaliar o postponement}

As empresas do segmento de sucos e conservas de frutas investigadas não possuem sistemas estruturados para medição de desempenho e não avaliam a estratégia de postponement. Existem acompanhamentos de algumas atividades produtivas e logísticas através de relatórios ou indicadores (medidas), mas não existe uma estruturação dessas medidas e nenhuma dessas é usada para medir especificamente o postponement. Apesar disso, os entrevistados indicaram medidas que consideram relevantes para avaliar o desempenho e possíveis resultados obtidos pela adoção do postponement. Essas medidas são apresentadas nos Quadro 7 a seguir. Adicionalmente, são destacados o número de empresas processadoras de sucos e produtoras de derivados de tomate que apontaram cada medida, bem como o somatório total no segmento, possibilitando assim, verificar quais as medidas de desempenho foram mais citadas por estas empresas. É importante também ressaltar que as medidas para avaliar a estratégia de postponement destacadas em azul são medidas indicadas pelos entrevistados da empresas investigadas que não foram destacadas no Quadro 5 apresentado no referencial teórico (medidas de desempenho para avaliar o postponement propostas por Zang e Tan (2001). Estas são medidas adicionais encontradas na pesquisa empírica. 
Quadro 7 - Medidas de desempenho de postponement nas empresas de sucos e conservas de frutas

\begin{tabular}{|c|c|c|c|c|}
\hline TIPO & MEDIDAS & $\begin{array}{c}\text { EMPRESAS } \\
\text { DE SUCO }\end{array}$ & $\begin{array}{l}\text { EMPRESAS } \\
\text { DE TOMATE }\end{array}$ & TOTAL \\
\hline \multirow[t]{4}{*}{ Custo Total } & Custo total & 3 & 2 & 5 \\
\hline & Custo por unidade & 3 & 3 & 6 \\
\hline & Custo total como percentual de vendas & 1 & 1 & 2 \\
\hline & Rentabilidade direta do produto & 2 & 1 & 3 \\
\hline \multirow{12}{*}{$\begin{array}{l}\text { Custos } \\
\text { Funcionais }\end{array}$} & Custo de transporte & 3 & 3 & 6 \\
\hline & Custo de armazenagem & 3 & 2 & 5 \\
\hline & Custo no processo de rotulagem & 3 & 3 & 6 \\
\hline & Custo no processo empacotamento & 3 & 3 & 6 \\
\hline & Custo no processo de montagem & 0 & 3 & 3 \\
\hline & Custo no processo de manufatura & 3 & 3 & 6 \\
\hline & Processamento de pedidos & 0 & 2 & 2 \\
\hline & Custo reverso & 0 & 0 & 0 \\
\hline & Custo de matéria-prima & 2 & 1 & 3 \\
\hline & Custo de mão-de-obra & 2 & 3 & 5 \\
\hline & Custo das mercadorias devolvidas & 1 & 1 & 2 \\
\hline & Valor real versus orçado de cada custo & 3 & 2 & 5 \\
\hline \multirow{6}{*}{ Gestão de ativos } & Giro de estoque & 2 & 3 & 5 \\
\hline & Custo de manutenção de estoque & 3 & 1 & 4 \\
\hline & Nível de estoque & 3 & 2 & 5 \\
\hline & Obsolescência & 2 & 3 & 5 \\
\hline & Retorno de investimento & 2 & 0 & 2 \\
\hline & Rentabilidade líquida de ativos & 2 & 0 & 2 \\
\hline \multirow{6}{*}{$\begin{array}{l}\text { Produtividade no } \\
\text { nível micro }\end{array}$} & Produtividade da mão-de-obra do armazém & 2 & 2 & 4 \\
\hline & $\begin{array}{l}\text { Unidades por unidade monetária de mão-de- } \\
\text { obra }\end{array}$ & 1 & 0 & 1 \\
\hline & Produtividade da mão-de-obra do transporte & 2 & 2 & 4 \\
\hline & Número de itens produzidos & 2 & 2 & 4 \\
\hline & Lead time da produção & 2 & 2 & 4 \\
\hline & $\begin{array}{l}\text { Tempo requerido para produzir um item } \\
\text { particular ou um conjunto de itens }\end{array}$ & 2 & 2 & 4 \\
\hline \multirow{2}{*}{$\begin{array}{l}\text { Produtividade no } \\
\text { nível macro }\end{array}$} & $\begin{array}{l}\text { Total de despesas operacionais/valor total das } \\
\text { mercadorias processadas }\end{array}$ & 2 & 2 & 4 \\
\hline & $\begin{array}{l}\text { Receita de Vendas - valor consumido na } \\
\text { operação }\end{array}$ & 1 & 1 & 2 \\
\hline \multirow{16}{*}{$\begin{array}{l}\text { Serviço ao } \\
\text { Cliente }\end{array}$} & Taxa de entrega & 1 & 1 & 2 \\
\hline & Porcentagem de falta de estoque por item & 1 & 3 & 4 \\
\hline & Taxa de entrega no tempo & 2 & 2 & 4 \\
\hline & Tempo de atendimento de pedidos pendentes & 1 & 2 & 3 \\
\hline & Lead Time total & 2 & 2 & 4 \\
\hline & Porcentagem de pedidos completos & 1 & 3 & 4 \\
\hline & $\begin{array}{l}\text { Tempo médio decorrido em cada atividade } \\
\text { envolvida no ciclo do pedido }\end{array}$ & 1 & 1 & 2 \\
\hline & Consistência do ciclo do pedido & 1 & 2 & 3 \\
\hline & Tempo de atraso médio & 1 & 3 & 4 \\
\hline & $\begin{array}{l}\text { Esforço para alterar de pedidos e habilidade da } \\
\text { empresa em atender as solicitações }\end{array}$ & 2 & 0 & 2 \\
\hline & $\begin{array}{l}\text { Porcentagem de pedidos que resultam em } \\
\text { reclamação }\end{array}$ & 2 & 2 & 4 \\
\hline & Custo incorrido para correção dos problemas & 1 & 1 & 2 \\
\hline & Motivos de reclamação & 2 & 2 & 4 \\
\hline & Tempo para resolução de problemas & 2 & 2 & 4 \\
\hline & $\begin{array}{l}\text { Informação da data de entrega no momento da } \\
\text { colocação do pedido }\end{array}$ & 2 & 2 & 4 \\
\hline & Integridade da mercadoria & 1 & 1 & 2 \\
\hline
\end{tabular}

Fonte: Elaborado pelas autoras com base nas entrevistas.

Analisando o Quadro 7 foi possível verificar que muitas das medidas destacadas na literatura por Zang e Tang (2001) para avaliar a estratégia de 
postponement foram também destacadas na pesquisa empírica como potenciais medidas para avaliar esta estratégia. Dentre as medidas comuns na literatura e prática, as mais citadas estão relacionadas à: logística (custos de transporte, armazenagem, manutenção de estoques); atividades operacionais que ocorrem mais à jusante da cadeia de suprimentos (manufatura final, empacotamento, montagem, rótulo e algumas medidas de serviço ao cliente (porcentagem de falta de estoque por item, taxa de entrega no tempo, entre outras).

As medidas logísticas foram bastante citadas, pois no caso das empresas investigadas, a elaboração de produtos semi-acabados possibilita uma mudança em custos logísticos, como os de transporte e estoque. No caso das empresas processadoras de suco de laranja, por exemplo, a aplicação da estratégia de postponement permite que o SLCC possa ser transportado com um volume bem menor que do produto final (suco pronto para beber), ocupando espaço muito menor nos armazéns.

As medidas associadas às atividades manufatura final, empacotamento, montagem, rótulo estão também entre as mais citadas, pois no caso das empresas de sucos e conservas de frutas, a postergação ocorre mais à jusante na cadeia de suprimentos. No caso das empresas processadoras de suco de laranja, a maior parte dessas atividades é postergada para outros membros da cadeia de suprimentos (empresas engarrafadoras de suco), e no caso das empresas de derivados de tomate, essas atividades são postergadas para o momento que a demanda é mais conhecida, mas acontecem internamente nas próprias empresas produtoras de derivados do tomate. Além disso, Bowersox e Closs (1996) destacaram que o postponement de forma pode ser sub-classificado em diferentes tipos, ou seja, pode ocorrer em diferentes atividades como: fabricação, embalagem, etiquetagem/rotulagem. Com exceção da atividade de montagem, que não ocorre nas empresas investigadas, o postponement de forma foi verificado nas outras atividades nas empresas pesquisadas, por isso, as medidas associadas às atividades manufatura final, empacotamento, montagem, rótulo estão também entre as mais citadas, e devem ser avaliadas.

Além das medidas já destacadas na literatura, os entrevistados indicaram medidas nas dimensões produtividade no nível macro e micro como potenciais 
medidas para avaliar o postponement, uma vez que esta estratégia também acarreta em mudanças na forma de fabricar o produto. Medidas para avaliar o postponement nestas dimensões ainda não haviam sido destacadas na bibliografia pesquisada. Adicionalmente, a formulação de produtos base (estado semi-acabado) pode agilizar a configuração final dos produtos assim que a demanda é conhecida. Ao mesmo tempo, a postergação pode aumentar os custos de produção devido ao fato da produção não ser contínua e da necessidade de re-processamento dos produtos. Por isso, essas medidas devem ser avaliadas.

Finalmente, é possível verificar no Quadro 7 que durante a pesquisa empírica surgiram diversas outras medidas na dimensão de serviço ao cliente, além das já destacadas na revisão de literatura. Segundo os entrevistados, a estratégia de postponement permite oferecer uma maior variedade de produtos em menor tempo, tendo assim, impacto direto no serviço oferecido aos clientes. Assim, medidas de desempenho para avaliar o postponement nestas dimensões devem ser identificadas e adotadas.

\section{CONSIDERAÇÕES FINAIS}

Este artigo apresentou uma discussão sobre medidas de desempenho para avaliar o postponement, onde foram identificadas na literatura e na pesquisa empírica quais as possíveis medidas de desempenho seriam adequadas para avaliar esta estratégia. Estas medidas foram identificadas e agrupadas nas dimensões custos, gestão de ativos, produtividade e serviço ao cliente. Para tanto, foram investigadas seis empresas do segmento de sucos e conservas de frutas, sendo três empresas processadoras de suco de laranja e três empresas que têm como principais produtos os derivados do tomate.

Os postponement de forma e tempo foram verificados nas seis empresas investigadas. Porém, em relação ao postponement de forma, este ocorre em diferentes locais da cadeia de suprimentos para as empresas processadoras de suco de laranja e empresa de derivados de tomate. Nas empresas processadoras de suco de laranja (S1, S2 e S3), as atividades de manufatura final, adição de embalagem, rótulo e distribuição são postergadas para outros membros da cadeia de suprimento, que em sua maior parte são empresas engarrafadoras localizadas 
em diferentes países no exterior. Já para as empresas de derivados de tomate, essas atividades são adiadas para o momento que a demanda é mais conhecida, mas são realizadas internamente pelas próprias empresas T1, T2 e T3.

Em relação à medição de desempenho, nenhuma das empresas de sucos e conservas de

frutas pesquisadas possui sistemas estruturados para medição de desempenho. Conforme já mencionado, algumas dessas empresas têm iniciado um processo de cadastramento de medidas de desempenho, avaliação e controle através de sistemas informatizados, mas não existe uma estruturação dessas medidas e nenhuma dessas é usada para medir especificamente o postponement. Alguns dos motivos são: o postponement foi implantado desde a fundação das empresas; e os entrevistados não consideram a adoção dessa estratégia um diferencial competitivo e sim, uma questão de sobrevivência, uma vez que os principais concorrentes também a adotam.

Entre as medidas mais adequadas para avaliar o postponement, as mais indicadas estão relacionadas à: logística (custos de transporte, armazenagem, manutenção de estoques); atividades que ocorrem mais à jusante da cadeia de suprimentos (manufatura final, empacotamento, montagem, rótulo); medidas de produtividade e medidas de serviço ao cliente (exemplo, disponibilidade e rapidez no atendimento ao cliente). Segundo os entrevistados, estas são as atividades mais sujeitas a modificações, caso a estratégia seja adotada.

Considera-se que o objetivo foi atingido, pois as medidas elencadas na literatura e na prática das empresas constituem um conjunto de medidas de desempenho importantes para avaliar $o$ desempenho da estratégia de postponement. O uso dessas medidas deve ser incentivado nas empresas de modo a melhorar os mecanismos de controle.

Finalmente, cabe ressaltar que pesquisas futuras devem ser realizadas buscando aplicação e complementação dos resultados. Este trabalho teve como objetivo identificar as diferentes formas de aplicação do postponement no setor alimentício e identificar possíveis medidas de desempenho que poderiam ser adotadas para avaliação desta estratégia. Assim, a efetividade do uso destas medidas não foi objeto de estudo, ou seja, não foi possível avaliar o quão bem o 
postponement vem sendo aplicado por essas empresas ao analisar o desempenho das mesmas nas medidas e perspectivas de desempenho sugeridas nesta pesquisa. Embora não sendo objeto de estudo deste trabalho, foi possível verificar que essa efetividade não poderia ser analisada nas empresas investigadas, uma vez que conforme já destacado, estas adotaram o postponement para se manterem no mercado, não se preocupando ainda em medir seu resultado.

Nesse sentido, pesquisas futuras neste setor devem buscar inicialmente propor a aplicação das medidas elencadas e sugeridas nesta pesquisa, para, posteriormente, verificar seus resultados efetivos, bem como a melhoria contínua do quadro de medidas sistematizado nesta pesquisa. Espera-se também que as medidas identificadas neste trabalho possam servir como base para elaboração de futuros sistemas de medição de desempenho para avaliação do postponement nas empresas.

\section{REFERÊNCIAS}

ALDERSON, W. Marketing efficiency and the principle of postponement. Cost and profit outlook, n.3, p. 15-18, 1950.

BEAMON, B.M. Measuring supply chain performance. International Journal of Operation and Production Management, v.19, n. 3, p. 275-292, 1999.

BOWERSOX, D. J., CLOSS, D J. Logistical management: the integreted supply chain process. New York, NY: McGraw-Hill, 1996.

BUCKLIN, L.P. Postponement, speculation and the structure of distribution channels. Journal of Marketing Research, v.2, p. 26-31, 1965.

CARDOSO, P. A. O princípio da postergação: um estudo na cadeia de suprimentos das tintas para impressão. 2002. $158 \mathrm{f}$. Tese (Doutorado em Engenharia de Produção) - Departamento de Engenharia Industrial da Pontíficia Universidade Catolica do Rio de Janeiro, Rio de Janeiro, 2002.

CLASSIFICAÇÃO NACIONAL DE ATIVIDADES ECONÔMICAS. Tabela de códigos e classificações. 2010. Disponível em: < http://www.cnae.ibge.gov.br>. Acesso em: ago. 2010.

CHOW, G.; HEAVER, T. D.; HENRIKSSON, L. E.. Logistics performance: definition and measurement. International Journal of Physical Distribution \& Logistics Management, v. 24, n.1, p. 17-28, 1994.

FAÉ, C.S. Análise crítica da colaboração em cadeias de suprimentos: o caso da cadeia automotiva gaúcha, 125 f., 2007. Dissertação (Mestrado em Engenharia Revista Produção Online. Florianópolis, SC, v.11, n. 2, p. 418-446, abr./jun., 2011 
de Produção e Transportes). Programa de Mestrado em Engenharia de Produção e Transportes, Escola de Engenharia, Universidade Federal do Rio Grande do Sul, Porto Alegre, 2007.

FERREIRA, K. A. Impactos do EDI e da internet na logística de empresas da indústria de alimentos. 2005. 180p. Dissertação (Mestrado em Engenharia de Produção) - Departamento de Engenharia de Produção da Universidade Federal de São Carlos, São Carlos, 2005.

FERREIRA, K. A.; ALCÂNTARA, R.L.; TOLEDO, J.C, O uso do postponement no desenvolvimento de produtos. In: SIMPÓSIO DE ENGENHARIA DE PRODUÇÃO, 13., 2006, Bauru. Anais eletrônicos... Disponível em:

<http://www.simpep.feb.unesp.br/simpep2007/upload/729.pdf>. Acesso em: nov.2006.

GHALAYINI, A. M.; NOBLE, J. S. The changing basis of performance measurement. International Journal of Operations \& Production Management, v. 16, n. 8, p. 6380, 1996.

GARCIA-DASTUGUE, S.; LAMBERT, D. Interorganizational time-based postponement in the supply chain. Journal of Business Logistics, v.28, n.1, p.5776, 2007.

GUNASEKARAN, A.; PATEL; C.; TIRTIROGLU, E. Performance measures and metrics in a supply chain environment. International Journal of Operations \& production management, v. 21, n. 1/2, p.71-87, 2001.

HIJJAR, M.F; GERVÁSIO, M.H.; FIGUEIREDO, K.F. Mensuração de desempenho logístico e o modelo World Class Logistics. (Partes 1 e 2). Artigos do Centro de Estudos em Logística. COPPEAD, UFRJ, 2005. Disponível em: < http://www.cel.coppead.ufrj.br/fs-busca.htm?fr-mensuracao_desempenho_1.htm>. Acesso em: jan. 2006.

LAMBERT, D.M; STOCK, J.R.; VANTINE,J.G. Administração estratégica da logística. São Paulo: Vantine Consultoria, 1998.

LIMA JR., O. F. Análise e avaliação do desempenho dos serviços de transporte de carga. In: CAIXETA-FILHO, J. V.; MARTINS, R. S. (ed.). Gestão logística do transporte de cargas. São Paulo: Atlas, 2001. p.108-147.

LEE, H. L.; TANG, C. S. Modelling the costs and benefits of delayed product differentiation. Management Science, v. 43, n.1, p.40- 53, 1997.

LEE, H.L.; BILLINGTON, C. Designing products and process for postponement. In: DASU, E.; EASTMAN, C. (Ed.). Management of design: engineering and management perspectives. Boston: Kluwer Academic Publishers, 1994. p. 105-122.

MERGULHÃO, R. C. Influência da medição de desempenho nos projetos seis sigma: estudos de caso. Tese (Doutorado em Engenharia de Produção), 
Departamento de Engenharia de Produção. São Carlos: Universidade Federal de São Carlos, 2007.

NEELY, A.; GREGORY, M.; PLATTS, K., Performance measurement system design: a literature review and research agenda. International Journal of Operations \& Production Management, v. 25, n. 12, p. 1828-1863, 2005.

NEELY, A.; AUSTIN, R. Measuring operations performance: past, present and future. In: NEELY, A. (Ed.). Performance measurement: past, present and future. Cranfield: Centre for Business Performance, 2000, p. 419-426.

NEELY, A. Measuring business performance. London: The Economist Books, 1998.

MONDRAGON, A.E.C; LALWANI, C.; MONDRAGON, C.E.C. Measures for auditing performance and integration in closed-loop supply chains. Supply chain management: an international journal, v.16, n.1, 2011, p.43-56.

PAGH, J.D.; COOPER, M.C. Postponement and speculation strategies: how to choose the right strategy. Journal of Business Logistics, v.19, n.2, p.13-32, 1998.

PIRES, S.R.I. Gestão da cadeia de suprimentos: conceitos, estratégias, práticas e casos - supply chain management. 2. ed. São Paulo: Atlas, 2009.

QUINTÃO, R.T.; Avaliação do desempenho logístico da cadeia brasileira de suprimentos de refrigerantes. 2003. 210 f.Dissertação (Mestrado em Engenharia de Produção). Departamento de Engenharia de Produção da Universidade Federal de Minas Gerais, Minas Gerais, 2003.

SAAD,M.; PATEL, B. An investigation of supply chain performance measurement in the Indian automotive sector. Benchmarking: An International Journal, v.13 n. 1/2, p. 36-53, 2006.

SELLITTO, M.A.; MENDES, L.W. Avaliação comparativa do desempenho de três cadeias de suprimentos em manufatura. Revista Produção, v.16, n.3, p.413-428, 2006.

SHEPHERD, C.; GÜNTER, H. Measuring supply chain performance: current research and future directions. International Journal of Productivity and Performance Management, v.55, n.3/4, p. 242-258, 2005.

VAN HOEK, R.I. . Postponement and the reconfiguration challenge for food supply chains. Supply Chain Management, v. 4, n.1, 1999, p.18-34.

YANG, B.; BURNS, N.D.; BACKHOUSE, C. J. Postponement: review and an integrated framework. International Journal of Operations \& Production Management, v.24, n.5, p.268-487, 2004.

YIN, R. K. Estudo de caso: planejamento e métodos. 2.ed. Porto Alegre: Bookman, 2001. 
ZHANG, C.; TAN, G. Classification of postponement strategies and performance metrics framework. In: PACIFIC ÁSIA CONFERENCE ON INFORMATION SYSTEMS, 5., 2001. Proceedings $\ldots$ Soul, Korea, 20-22 june, 2001. 3 em: < http://www.pacis-net.org/file/2001/038.PDF>.

ZINN, W.; BOWERSOX, D.J. Planning physical distribution with the principle of postponement. Journal of Business Logistics, v.9, n.2, p.117-136, 1988.

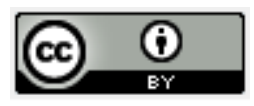

Artigo recebido em 15/04/2010 e aceito para publicação em 10/04/2011. 